\title{
Maßnahmen gegen Selbstbediener
}

\begin{abstract}
Kaum ein Tag vergeht, an dem nicht von neven Skandalen aus Unternehmen berichtet wird. 'Frisierte Bilanzen' und 'Selbstbedienung von Managern' scheinen Zeichen eines allgemeinen Niedergangs der Moral zu sein. Wer jedoch nicht auf der Ebene allgemeiner moralischer Empörung stehen bleiben will, muss sich fragen, was diese Fehlhandlungen ermöglicht und warum Risikomanagement versagt. Ein umfassendes und integratives Wertemanagement-System kann dazu beitragen, dass Grundsätze nicht nur auf dem Papier stehen, sondern zu einem Standard verantwortungsvoller Unternehmensführung werden.
\end{abstract}

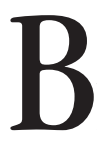
Von Josef Wieland islang wird "Corporate Governance" allgemein als Steuerungsstruktur zur Abwicklung wirtschaftlicher Transaktionen in, zwischen und mittels Unternehmen definiert. Die Steuerungsstruktur setzt sich aus formalen und informalen Regeln sowie aus organisatorischen Einrichtungen zur Führung und Kontrolle eines Unternehmens zusammen.

Im Interesse einer verantwortungsvollen Unternehmensführung sollte die momentane Verengung von Corporate Governance auf die rechtliche Seite der Unternehmensfïhrung oder auf die Beziehung Vorstand - Aufsichtsrat jedoch für ökonomische, soziale oder ökologische Aspekte geöffnet werden. Ein Schritt in diese Richtung wurde mit dem Deutschen Corporate Governance-Kodex (DCGK) unternommen. Dieser beinhaltet neben Gesetzestreue auch ,Standards guter und verantwortungsvoller Unternehmensführung“. Der Vorstand ist sowohl an das Unternehmensinteresse gebunden, als auch ausdrücklich auf die „Steigerung des nachhaltigen Unternehmenswertes" verpflichtet.

Man kann davon ausgehen, dass der Begriff der Nachhaltigkeit dort nicht ohne Grund steht. Im DCGK läuft somit eine potenzielle StakeholderOrientierung mit. Zwar geht es auch hier um bessere Kontrollrechte der Aktionäre, diese Compliance-Aspekte sind jedoch in das Gesamt der Unternehmensfiuhrung eingebettet. Punkt 4 des Kodex versteht darunter die strategische Ausrichtung des Unternehmens, seine Ausrichtung an den Gesetzen sowie wirkungsvolles Risikomanagement und Risikocontrolling.

Die im Deutschen Corporate Governance-Kodex festgeschriebenen Verhaltenstandards für Ziele und Aufgaben des Vorstandes sind als Ergän- zungen der gesetzlichen Vorschriften konzipiert. Sie sind „soft law", für dessen Durchsetzung es der Selbstbindung der Akteure bedarf. Ohne Loyalität, Integrität und Ehrlichkeit des Vorstandes sowie aller Mitarbeiter gegenüber dem Unternehmen ist das nicht zu haben. „Soft law“ setzt zu seiner Wirksamkeit die moralische Selbstbindung der beteiligten organisationalen und individuellen Akteure voraus.

\section{Risikomanagement und Verhaltensrisiken}

Gerade die Bilanzfälschungsskandale der letzten Jahre, Enron, WorldCom e tutti quanti, haben gezeigt, wie eine enge Shareholder Value-Perspektive und eine entsprechende Entgeltpolitik für Topmanager geradezu ideale Anreize für alle Arten doloser Handlungen bieten. Kurzfristige Gewinnerwartung der Aktionäre, Einkommen maximierende Strategien des Topmanagements und die langfristige Sicherung der Unternehmensexistenz lassen sich nur durch ein umfassendes Risikomanagement verbinden. Neben die bekannten Risiken aus Geschäften und Prozessen treten Risiken aus Verhalten, auf die ein Risikomanagement sensibel und präventiv reagieren muss. Die herkömmlichen Risikomanagementsysteme blenden diesen Aspekt aber aus.

Die oben erwähnten Unternehmen waren alle mit beispielhaften Compliance-Programmen ausgestattet. Aber die sind nur die Papiervoraussetzung dafür, worum es im Kern geht: Wie mobilisiert ein Unternehmen systematisch und nachhaltig ein rechtlich und moralisch integres Verhalten von Führung und Mitarbeitern? Wie schafft es Anreize, dass die Regelungen der Corporate Governance gelebt werden? Wie eliminiert es betriebliche Anreize, die illegales und unmoralisches Verhalten prämieren? Corporate Governance und Risikomanagementsysteme, die ausschließlich ,law driven“ sind, sind hierfür zwar notwendig, aber nicht ausreichend. Darüber hinaus braucht es die moralische Verpflichtung einer Organisation und ihrer Mitglieder auf die Erfïllung solcher Spielregeln, und diese kann nur ,value driven“ sein.

\section{- Wertemanagement als Risikoprävention}

Werte und moralische Vorstellungen sind handlungs- und verhaltenssteuernde informale Institutionen. Die Frage ist daher nicht, $o b$ Organisationen und ihre Mitglieder über Werte und Moral verfügen, sondern über welche. Die Handlungen von Mitgliedern einer Organisation lassen sich demnach nicht allein durch Direktion und Kontrolle, durch Anreize und Sanktionen, sondern grundlegend auch durch Werte - Einstellungen, Haltungen, Überzeugungen - steuern. Sie sind informale Governancestrukturen jeder Unternehmung. Ich habe dafür den Begriff der Governanceethik vorgeschlagen, der deutlich machen soll, dass die Frage von Werten der Moral nicht in den Bereich des good will eines Unternehmens gehört, sondern unabtrennbarer Bestandteil der Steuerung und Kontrolle von Unternehmen ist. Aus der Sicht der Governanceethik stellt sich nicht die Frage, ob ein Unternehmen sich mit seinen Werten beschäftigt oder nicht, sondern allein, ob diese Seite unternehmerischer Entscheidungen gezielten Managementanstrengungen unterworfen wird. Gerade in Bereichen, in denen die Regeleffizienz und Regeleffektivität formaler Institutionen wie Recht und kodifizierte Verhaltensstandards mit der zunehmenden Komplexität von Managemententscheidungen abnimmt, müssen die moralischen Ressourcen einer Organisation und ihrer Mitglieder mobilisiert werden.

Wer genau jene Probleme eindämmen will, die zu der gegenwärtigen Corporate Governance-Bewegung geführt haben und allesamt ihren Ursprung in Grauzonen, Informationsasymmetrien oder unüberwindbaren Kontrolldefiziten nehmen, sollte ein Wertemanagementsystem als Bestandteil seiner Corporate Governance implementieren. Ein am Konstanz Institut für WerteManagement (KIeM) gerade abgeschlossenes Forschungsprojekt zum werteorientierten Risikomanagement hat gezeigt, dass moralisch gesteuerte Risikosensibilisierung und Risikoprävention grundlegende Elemente eines Risikomanagementsystems sind, das nicht nur Risiken aus Geschäften und Prozessen, sondern vor allem aus Verhalten absorbieren 
kann. Die Studie zeigt, dass Werte Risikowahrnehmungen und damit deren Relevanz und risikorelevantes Verhalten mitsteuern. Es geht um vollständige Risikoerfassung und um die Kontrolle der entsprechenden Risikoindikatoren sowie die Schaffung einer Wertekultur, die Risiken von Anfang an eindämmt.

Zu diesem Zweck haben wir am KIeM in den letzen Jahren ein vierstufiges Wertemanagementsystem entwickelt und in einer ganzen Reihe von Unternehmen und Branchen eingeführt. Anfang dieses Jahres wurde es in Zusammenarbeit mit zu einem Standard weiterentwickelt, der Mindestanforderungen für ein solches Wertemanagementsystem enthält. Unternehmen wie Siemens, ABB, BASF, Novartis, Schwarz Pharma, Fraport, Yello Strom, Thyssen

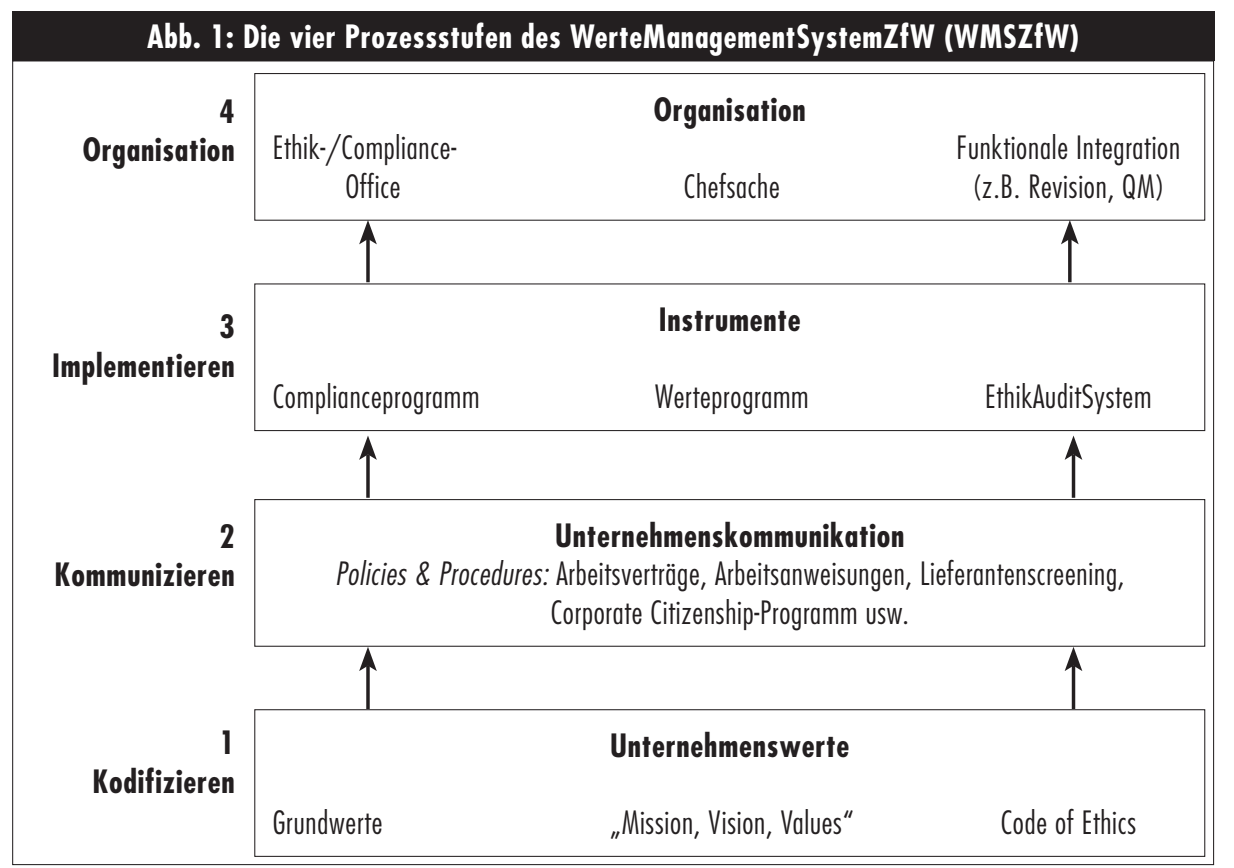

\section{- Prozessstufen des Wertemanagements}

Das WerteManagementSystem beginnt mit der Kodifizierung der Grundwerte eines Unternehmens, die klar und lebbar sein müssen. Hier entscheidet sich die Identität einer Organisation anhand der Frage, welche Art von Geschäften ein Unternehmen überhaupt machen will. Auf der nächsten Ebene müssen diese Grundsätze intern und extern kommuniziert werden. Diese Ebene ist die entscheidende, da hier die Grundwerte praktisch umgesetzt werden. Dazu müssen sie in alle Arbeitsanweisungen, Arbeitsprozesse und Leitlinien eingebaut werden. Training und Seminare leisten dazu einen grundlegenden Beitrag, reichen aber nicht aus. Dies gilt analog für die interne und externe Kommunikation. Einbezogen werden müssen alle potenziell sensiblen Bereiche, also etwa Karriereplanungen, Entgeltpolitik, Zielvereinbarungen, Lieferantenbewertungen, Umgang mit Geschenken und so weiter. Anforderungen an die Werthaltungen von Mitarbeitern lassen sich zum Beispiel in Karriereplanungen in der Form einbauen, dass niemand in Führungspositionen aufrückt, der diese Kriterien nicht erfüllt.

Auf der dritten Ebene müssen die einzelnen Maßnahmen und Instrumente so aufeinander abgestimmt werden, dass sie ein möglichst konfliktfreies Managementsystem ergeben. So ist es etwa ein Widerspruch, Entlohnungs- und Bonisysteme strikt ertrags- oder umsatzorientiert zu gestalten und gleichzeitig integres Verhalten in schwierigen,

Quelle: Eigene Darstellung

nordamerikanischen Kontext eher Ethics Officers eine wichtige Rolle spielen, scheint sich in Deutschland die funktionale Integration dieser Programme durchzusetzen, etwa in das Qualitätsmanagement, die Kommunikationsabteilung oder eine Stabstelle. Beide Varianten können nur Wirkung entfalten und lebensfähig sein, wenn sie durch das offensive und glaubwürdige Engagement der Unternehmensleitung gestützt werden.

\section{$>$ Fazit}

Die Wirkung von Corporate Governance-Programmen hängt davon $a b$, wie umfassend und integrativ sie angelegt sind. Ihre steuernde Funktion basiert nicht allein auf formalen Regelungen, sondern darauf, wie diese über eine Mobilisierung der informalen Ressourcen einer Organisation tatsächlich mit Leben erfïllt werden. Es sind somit die moralischen Grundlagen der Corporate Governance, die über die Qualität der Führung und Kontrolle eines Unternehmens mit entscheiden. Erst die Transformation der Werte von einem individuellen zu einem organisationalen Anliegen mittels eines WerteManagementSystems sorgt dafür, dass ein lebendiger Prozess entstehen kann. Dabei muss klar sein, dass es sich um einen Prozess handelt und nicht um die Einführung von Moral per Stichtag. Der Lernprozess der Organisation und ihrer Mitglieder zielt auf den Erwerb der Kompetenz ab, den unvermeidbar moralischen Aspekt wirtschaftlicher Transaktionen zu erkennen und so zu gestalten, dass er einen positiven Beitrag zur Sicherung des Unternehmens in ökonomischer, juristischer und gesellschaftlicher Hinsicht leistet. Wertemanagementsysteme sind selbst ein „Standard guter und verantwortungsvoller Unternehmensführung“ und dienen der „Steigerung des nachhaltigen Unternehmenswertes“ (DCGK).

\section{Literatur}

Wieland, J.: Die Ethik der Governance, Marburg 1999. Wieland, J.: Corporate Governance und Wertemanagement. In: Mittelstraß, J. (Hg.): Die Zukunft der Wissensgesellschaft, Berlin 2000

Wieland, J.: Eine Theorie der Governanceethik. In: Zeitschrift für Wirtschafts- und Unternehmensethik, Jahrgang 2, Heft 1, 2001, S. 8-33.

Wertemanagements und evaluieren die Existenz und Wirksamkeit des implementierten Werteprogramms durch Dokumentation und Befragung entweder als Fremdsteuerungssystem durch unternehmensexterne Gruppen oder als Selbststeuerungssystem eines Unternehmens mit oder ohne externe Prüfung.

Auf der vierten Stufe geht es um die Organisation eines Wertemanagementsystems. Während im

\section{Der Autor}

Prof. Dr. Josef Wieland ist Direktor des (KleM) Konstanz Institut für WerteManagement Kontakt: KleM, Fachhochschule Konstanz, Brauneggerstr. 55, 78462 Konstanz, Tel. 07531-206404, E-Mail: wieland@fh-konstanz.de 
(c) 20I0 Authors; licensee IÖW and oekom verlag. This is an article distributed under the terms of the Creative Commons Attribution Non-Commercial No Derivates License (http://creativecommons.org/licenses/by-nc-nd/3.o/), which permits unrestricted use, distribution, and reproduction in any medium, provided the original work is properly cited. 